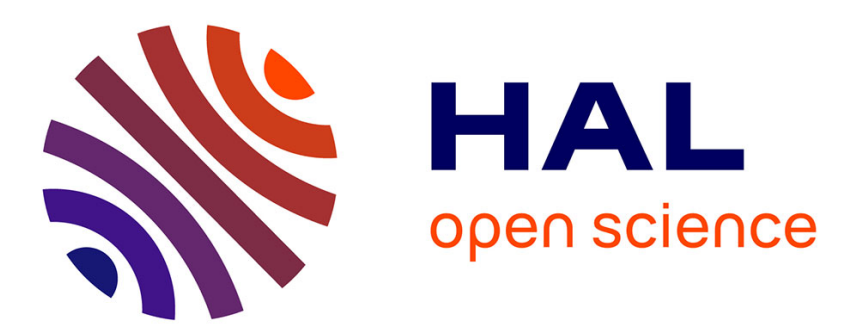

\title{
Une analyse discursive d'un type de reprise immédiate: la reprise autonymique, dans les énoncés définitoires
} Josette Rebeyrolle

\section{To cite this version:}

Josette Rebeyrolle. Une analyse discursive d'un type de reprise immédiate: la reprise autonymique, dans les énoncés définitoires. Journal of French Language Studies, 2005, 15, pp.67-82. halshs00420631

\section{HAL Id: halshs-00420631 https://shs.hal.science/halshs-00420631}

Submitted on 29 Sep 2009

HAL is a multi-disciplinary open access archive for the deposit and dissemination of scientific research documents, whether they are published or not. The documents may come from teaching and research institutions in France or abroad, or from public or private research centers.
L'archive ouverte pluridisciplinaire HAL, est destinée au dépôt et à la diffusion de documents scientifiques de niveau recherche, publiés ou non, émanant des établissements d'enseignement et de recherche français ou étrangers, des laboratoires publics ou privés. 
Rebeyrolle, J. (2005). Une analyse discursive d'un type de reprise immédiate : la reprise autonymique, dans les énoncés définitoires. Journal of French Language Studies, 15, 6782.

\section{Une analyse discursive d'un type de reprise immédiate : la reprise autonymique, dans les énoncés définitoires Josette Rebeyrolle* \\ Université de Toulouse-Le Mirail - CNRS \\ Equipe de Recherche en Syntaxe et Sémantique \\ Maison de la Recherche, 5 allées A. Machado \\ 31058 Toulouse cedex, France \\ rebeyrol@univ-tlse2.fr}

Reprenant à notre compte la distinction proposée par Riegel (1987, 1990) entre deux types d'énonciation définitoire, nous distinguons l'énonciation littérale d'un acte de langage définitoire, de l'énonciation indirecte servant à accomplir ce même acte. Cette distinction repose sur le fait que, dans le premier cas, l'intention du locuteur est explicitée, alors que, dans le second, elle est présentée indirectement, par un acte indirect. Si dans la première situation, la force illocutionnaire de l'énoncé est clairement indiquée par les éléments métalinguistiques qui servent à accomplir l'acte définitoire, dans la seconde, en revanche, le lecteur infère ce que le locuteur entend communiquer. Les structures métalinguistiques qui signalent le contenu définitoire de l'énoncé sont bien connues. Il s'agit des formes illustrées par les exemples construits suivants :

(1) On appelle "cormoran", un oiseau de mer à ailes imperméables.

(2) Le terme cormoran désigne un oiseau de mer à ailes imperméables.

(3) Un oiseau de mer à ailes imperméables s'appelle un cormoran.

De ces situations où le lecteur dispose d'indices qui déclenchent l'interprétation définitoire de l'énoncé, on distingue celles où aucun indice superficiel ne signale l'intention du scripteur. Notre objectif est donc ici de mettre au jour la manière dont se marque l'intention du locuteur dès lors que l'énoncé définitoire qu'il choisit prend la forme d'un énoncé qui ne comporte aucun indicateur explicite de la force illocutionnaire de l'énoncé. On s'intéresse ici à la structure syntaxique représentée par le schéma suivant : [SNa est un $\mathrm{Nx}-\mathrm{X}]$, illustrée par :

*Je tiens à remercier Francis Cornish pour ses précieuses remarques et suggestions qui m'ont aidé à améliorer ce texte. Les erreurs et mauvaises analyses éventuelles sont de mon fait. 
(4) $\left[\begin{array}{ll}\text { Un } & \text { cormoran }\end{array}\right]_{\mathrm{SNa}}$ [est un $]$ [oiseau de mer $]_{\mathrm{Nx}}$ [à ailes imperméables] $]_{\mathrm{X}}$.

Il s'agit d'une proposition assertive canonique de structure S Copule Attribut dont la syntaxe est non marquée puisque l'ordre linéaire de la phrase reflète l'ordre de l'information. Les variantes de cette structure ne seront pas examinées. Ainsi laissera-t-on de côté, des formes comme "un Nx - X est un $\mathrm{Na}$ " ou "un $\mathrm{Nx}$ - X c'est un $\mathrm{Na"} \mathrm{qui} \mathrm{n'ont} \mathrm{pas} \mathrm{la} \mathrm{même} \mathrm{structure}$ informationnelle puisque, comme cela a été maintes fois relevé, le placement des constituants sur l'axe syntagmatique a un effet sur le poids informationnel. Dans la structure qui retient notre attention ici, la position initiale est occupée par le sujet grammatical, $\mathrm{SNa}$, position intimement associée à la fonction de thème ou topique (quelle que soit la terminologie qu'on adopte, on considère généralement qu'il s'agit d'un élément informationnellement léger dont le référent, placé en début de phrase, est présenté comme accessible, supposé connu du lecteur, ce qui suppose qu'il a déjà été introduit ou qu'il est facilement accessible 'indirectement', autrement dit par le biais d'une inférence attendue de la part de l'allocutaire). La position finale, $[\mathrm{Nx}-\mathrm{X}]$, est quant à elle réservée au rhème, autrement dit à la partie la plus saillante, il s'agit d'un constituant exprimant le focus, c'est-àdire l'information nouvelle.

Nous cherchons ici à mettre au jour les éléments qui aident le lecteur à interpréter un énoncé de forme [SNa est un $\mathrm{Nx}-\mathrm{X}$ ] comme un énoncé définitoire. Il s'agira de répondre à la question suivante : comment le scripteur fait-il pour signaler à son lecteur son intention de définir alors que superficiellement l'énoncé qu'il produit ne comporte aucune trace de cette intention? Nous essayerons de repérer, dans le fil du discours, des éléments qui annoncent, qui préparent l'énonciation définitoire indirecte. Par conséquent, nous nous situerons à la fois sur le plan de la linéarité du texte et sur le plan informationnel. Chemin faisant, nous montrerons que l'analyse discursive fournit une justification complémentaire de la thèse du sens définitoire indirect défendue par Riegel (1987, 1990). 
Nous partons d'une observation fondée sur des données attestées ${ }^{1}$ : il existe un site textuel particulièrement favorable à l'insertion d'un énoncé définitoire indirect. Ce site se caractérise par la reprise immédiate de la première mention du terme à définir $(\mathrm{Na})$ au sein de l'énoncé définitoire indirect, comme l'illustre l'exemple suivant :

(5) Au loin, les marins virent passer un cormoran. Le cormoran est un oiseau de mer à ailes imperméables. [...]

Ce type d'enchaînement ayant peu retenu l'attention, à notre connaissance, nous avons choisi de l'étudier afin de mettre au jour les contraintes qui le régissent. Notre analyse se fera en deux temps. Après avoir montré le traitement que les études antérieures ont réservé à ce type d'enchaînement, nous examinerons l'hypothèse d'un décrochage autonymique pour expliquer ces situations discursives considérées comme marquées.

\section{Explications antérieures}

L'enchaînement qui nous intéresse ici et qu'illustre (5) ci-dessus, a été décrit par Combettes \& Tomassone (1988) comme une "progression linéaire", où le thème $\mathrm{T} 2$ fait partie du rhème $\mathrm{R} 1$ et $\mathrm{T} 3$ de $\mathrm{R} 2$, comme le montre la représentation suivante :

$$
[\mathrm{T} 1->\mathrm{R} 1]_{\mathrm{P} 1}->[\mathrm{T} 2->\mathrm{R} 2]_{\mathrm{P} 2}->[\mathrm{T} 3->\mathrm{R} 3]_{\mathrm{P} 3}
$$

Ce type d'enchaînement se caractérise par le fait qu'un constituant introduit sous une forme rhématique est repris comme thème dans le contexte qui suit, chaque thème étant "issu" du rhème de la phrase précédente. Les auteurs observent que ce type de progression thématique convient parfaitement aux textes explicatifs dont l'un des objectifs est d'approfondir

\footnotetext{
1 Les exemples sont principalement extraits de deux ouvrages: un manuel de géomorphologie : Derruau, M. (1998). Précis de géomorphologie, Paris : Masson et un recueil d'articles scientifiques : Charlet, J., Zacklad, M., Kassel, G \& Bourigault, D. (2000). Ingénierie des connaissances, évolutions récentes et nouveaux défis. Paris : Eyrolle. Les extraits empruntés au premier seront notés [Précis] et ceux tirés du second [IC].
} 
un phénomène par paliers successifs, le thème issu du rhème précédent introduisant une précision, un exemple ou un complément d'information.

Les auteurs ne fournissant pas d'exemple très clair de la progression linéaire, nous en emprunterons un à Riegel et al. (1994: 608) :

(6) Le fantôme se matérialise par le "simulacre du volume". Le simulacre du volume est l'enveloppe. L'enveloppe cache, protège, transfigure, invite, tente, donne une notion trompeuse du volume. (Salvador Dali)

A l'occasion d'un rappel de la typologie proposée par Combettes et Tomassone, Riegel et al. tentent de préciser les propriétés de la progression linéaire. L'analyse fournie par La grammaire méthodique du français ne nous semble toutefois pas satisfaisante. Nous allons essayer de préciser pourquoi. Les auteurs rappellent d'abord que, comme le montre Kleiber (1994: 103), le cas privilégié de l'anaphore est la substitution pronominale (les formes non marquées d'expression de la relation anaphorique étant les pronoms de troisième personne clitiques et les formes nulles, ou zéros). Ils interprètent le rôle du pronom comme un moyen d'éviter la répétition d'un nom puisqu'il marque la continuité thématique en signalant la maintenance du thème repris. Or, l'enchaînement qui fait la spécificité de la progression linéaire n'est pas compatible avec cette règle générale de coréférence. Cette incompatibilité ne trouve cependant pas d'explication chez Riegel et al. qui se contentent d'affirmer que : 'les rôles des pronoms dans la structuration du texte sont ... diversifiés' (ibidem, 163). La répétition de la dénomination de première mention pose un problème qui ne trouve pas ici de solution puisque l'anaphore pronominale demeure la forme la plus attendue.

Notre objectif ici est d'essayer d'aller plus loin et de mettre en évidence les contraintes qui régissent la réitération en P2 de R1 sous couvert de la dénomination de première mention. L'éclairage d'une théorie du discours devrait nous permettre de progresser dans ce but. Nous ferons un détour par la théorie du Centrage pour deux raisons. D'abord parce que cette théorie propose une description des enchaînements les plus probables et ensuite parce qu'elle cherche à prédire le degré de cohérence d'un segment de 
discours en tenant compte de la difficulté relative de son traitement par le locuteur.

\subsection{Continuité topicale}

La théorie du Centrage (Walker, Joshi \& Prince, 1998 ; Cornish, F. (éd) 2000) étant une théorie qui permet, selon nous ${ }^{2}$, de faire le lien entre la structure informationnelle des énoncés et la cohérence des segments de discours dans lesquels ils s'inscrivent, elle peut nous apporter des éléments de réponse pour expliquer la question de la répétition de la dénomination.

Selon cette théorie basée sur la topicalité des référents, la transition préférée est la continuation de l'entité le plus centralement concernée par P2 et qui fait le lien entre $\mathrm{P} 2$ et ce qui précède, à savoir P1. Cette transition, nommée continuation du centre rétroactif (désormais abrégé en $\mathrm{Cr}$ ) antérieur, prévoit qu'en $\mathrm{P} 2$, le $\mathrm{Cr}$ soit exprimé par un pronom sujet sélectionnant le référent le plus élevé à l'intérieur du centre anticipateur (désormais abrégé $\mathrm{Ca}$ ) de l'énoncé précédent. On trouvera donc par exemple de préférence le pronom il pour sélectionner le référent masculin le plus élevé à l'intérieur du Ca de P1. En effet, garder le même centre en P2 et P3 est considérée comme une continuation plus cohérente. La théorie préfère également la rétention par rapport au déplacement qui est de loin l'enchaînement considéré comme le moins souhaitable lorsqu'il s'effectue brutalement. En revanche, lorsqu'il s'opère en douceur, le déplacement reste un enchaînement acceptable.

Si l'on se conforme strictement aux prédictions de la théorie, on dira qu'un référent connu, accessible sera préférentiellement verbalisé par une forme pronominale ou nominale dont le contenu informatif est faible. Or, dans les structures étudiées ici, le référent connu est celui qui occupe la fonction sujet dans P1, comme le montre l'extrait suivant :

\footnotetext{
2 Bien que les théoriciens du Centrage s'en défendent, pour la plupart, dans la mesure où ils nient que la distribution des expressions indexicales dans les énoncés est régie par la structure informationnelle de la phrase énoncée. Pour une discussion sur les problèmes posés par la théorie du centrage, on se reportera notamment à Schnedecker (2003).
} 
(7) [Par exemple, il est facile de retrouver la conclusion d'un rapport parce que c'est la dernière section du rapport.] [La dernière section est celle qui se trouve à la fin du document physique.] [Une section se reconnaît par le fait qu'elle est composée de blocs de texte (les paragraphes) situés sous un titre $]_{\mathrm{P} 1}$. [Le titre est un bloc court isolé et typographié dans une fonte plus grasse, éventuellement souligné et numéroté $]_{\mathrm{P} 2}$. [IC]

Une reprise pronominale est possible mais uniquement sous la forme de $c e$ ou $c^{\prime}$ ou encore de il s'agit de. Cependant, ces formes ne sont pas aptes à reprendre le référent qui se trouve en position thématique dans P1. Dans cette situation, en effet, on les interprète plutôt comme référant au référent du SN en focus dans P1, car seul le sujet de P1 (une section) peut accepter d'être repris en P2 par une forme pronominale. C'est ce que montre la paraphrase suivante :

(8) [Une section se reconnait par le fait qu'elle est composée de blocs de texte (les paragraphes) situés sous un $\underline{\text { titre }}]_{\mathrm{P} 1}$. ['est une.... $]_{\mathrm{P} 2}$.

Dans la situation illustrée ici, la contrainte qui veut que le $\mathrm{Cr}$ de $\mathrm{P} 2$, dans une transition de type continuation, soit le membre classé premier du Ca est clairement enfreinte. On ne se trouve donc pas dans la situation par défaut de la continuité topicale. Par conséquent, les situations que nous décrivons seront considérées par la théorie comme peu cohérentes car elles enfreignent les règles qui, lorsqu'elles sont respectées, permettent un traitement cognitif aisé par l'auditeur. La réitération du même $\mathrm{N}$ est donc un facteur pénalisant pour cette théorie. Finalement, la théorie du Centrage expliquerait le fait que la reprise fidèle est sélectionnée en invoquant l'impossibilité d'une anaphore par c'est, celle-ci étant préférentiellement interprétée comme renvoyant au référent évoqué par l'emploi du SN une section qui constitue le topique.

On pourrait se demander si ce que l'on observe s'explique par une transition par déplacement en douceur. Reprenons les termes de la théorie. Selon le Centrage, on ne peut pas mettre en position sujet une entité qui n'a pas encore été introduite dans l'énoncé précédent, comme le montre, dans les exemples suivants empruntés à Cornish (2000), l'impossibilité d'un déplacement brutal en (12) : 
(9) Jean est un type sympa.

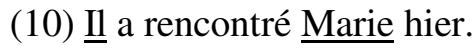

(11) Elle était avec Lucie.

(12) \#Lucie était avec elle.

Bien que, dans les énoncés du type de ceux illustrés par (7), l'entité réalisée en ei-1 occupe une position périphérique, la forme de reprise sélectionnée résiste à la théorie.

\subsection{Rôle du prédicat classifiant}

Dans son ouvrage consacré à la référence, aux pronoms, à l'anaphore et à la deixis, Kleiber (1994) essaie d'expliquer les problèmes d'acceptabilité que posent des exemples du type :

?Pussy, il est un angora.

dans lesquels le référent est rendu saillant par détachement. L'impossibilité d'une anaphore pronominale par il s'explique, selon l'auteur, par l'effet de deux exigences contradictoires : continuité $v s$ rupture. La première est que $i l$ exige une continuité qui se trouve bloquée par la présence d'un prédicat classifiant et la seconde que l'opération de catégorisation réalisée par le prédicat classifiant entraîne une rupture car elle a pour effet de recatégoriser le référent. La reprise du référent de première mention ne peut pas se faire avec $i l$ dans ce site en raison du fait que la reprise anaphorique exige une continuité que le prédicat classifiant ne peut établir puisqu'il a pour effet au contraire d'introduire une rupture.

Selon Kleiber, deux types de phrases ont toutefois la capacité de rétablir le nécessaire prolongement entre la phrase contenant il et la phrase impliquant le référent. Il s'agit d'abord de la qualification, dans des énoncés comme :

(13) Il est un critique merveilleux qui n'a pas suffisamment tiré partie de son talent (Boone, 1989).

Kleiber considère que $i l$ se justifie ici car la phrase fournit une description du référent et non pas une classification. 
Le second type de phrase qui autorise il est à chercher du côté des phrases identificationnelles qui admettent d'ailleurs aussi bien il que $c^{\prime}$ ou $c e$, comme on le voit dans :

(14) Max, il/c' est le directeur de la banque.

Ces deux situations sont considérées comme acceptables par Kleiber car dans les deux cas le prédicat introduit non plus une rupture mais une continuité.

En suivant Kleiber, on dira que c'est parce que il nécessite une continuité entre le référent de première mention, en $\mathrm{P} 1$, et sa reprise, en $\mathrm{P} 2$, qu'il est exclu qu'il occupe la position sujet d'un prédicat classifiant ayant pour fonction de (re)catégoriser le référent préalablement introduit. Nous trouverons dans les exemples fournis dans notre troisième section une confirmation du fait que $i l$ ne peut saisir un référent qui est destiné à être recatégorisé. Il n'est pas acceptable dans une phrase classifiante car ce type de phrase entraîne, comme le dit Kleiber (1994: 75), 'une sortie du cadre qui a rendu le référent de $i l$ manifeste'. On explique alors pourquoi $i l$ est exclu dans cette position en invoquant une rupture. Superficiellement P2 est un prolongement de $\mathrm{P} 1$, mais il s'agit uniquement d'un prolongement de surface. Le prédicat classifiant a pour rôle d'opérer une classification, autrement dit il assigne au référent une place au sein d'une catégorie, de ce fait il n'a pas la capacité d'attribuer au référent précédemment introduit une propriété spécifique. L'opération de classification qu'opère le prédicat est incompatible avec le prolongement qu'impose $i l$, autrement dit on peut classifier sans introduire une rupture. Cette analyse s'accorde avec notre intuition de l'introduction d'une rupture que nous avons analysée comme une rupture autonymique.

\subsection{Le changement de genre}

A l'occasion d'une analyse montrant que l'anaphore n'est pas un

phénomène purement syntaxique, mais un phénomène sémantico- 
grammatical, Cornish (1999: 46) insiste sur l'importance du prédicat qui gouverne l'anaphore. Dans ce but, il utilise des exemples qui attestent le changement de genre qui affecte, dans certaines situations, l'anaphore pronominale. Ce sont ces deux exemples que nous voudrions discuter. Commençons par rappeler ces deux extraits du journal Le Monde :

(15) La politique n'est pas le royaume de l'absolu, et ceux qui croient le contraire ont toujours connu d'effroyables réveils. Elle est un art, difficile comme tous les arts, mais qui doit, sauf à sombrer dans le chaos qu'il vise précisément à ordonner un peu, respecter un minimum de règles...

(16) L'homme est une espèce soumise aux règles biologiques comme les autres espèces. Elle a besoin pour vivre que l'ensemble de son environnement, la biosphère, soit harmonieusement équilibrée...

Cornish explique le passage en (15) du féminin (la politique) au masculin (il) et en (16) du masculin (l'homme) au féminin (elle) par le fait qu'un changement de perspective est introduit par le prédicat classifiant. Dans les deux cas, selon lui, la catégorie dans les termes de laquelle le référent (la politique, l'homme) est (re)conceptualisé (est un art ou est une espèce) est répétée dans le co-texte (difficile comme tous les arts ou comme les autres espèces) ce qui est une façon de signaler qu'un changement de perspective s'opère. Dans les deux exemples, c'est bien, comme le dit Cornish, la répétition de la catégorie qui conditionne le changement de perspective et qui justifie le changement de genre. Si l'on supprime la répétition (comme tous les arts, comme les autres espèces), le changement de point de vue disparait et consécutivement la forme du pronom conserve le genre du référent du SN (la politique, l'homme).

Ces exemples nous intéressent pour deux raisons en partie disjointes de celles qui ont conduit Cornish à les relever. (15) nous fournit une attestation de reprise pronominale avec un prédicat classifiant (elle est un art), énoncé que Kleiber ferait sans doute précéder d'un point d'interrogation. On peut toutefois expliquer cette anaphore pronominale en invoquant le rôle du parallélisme syntaxique qui caractérisent les deux phrases successives : n'est pas le ... royaume vs est un ... art. C'est ce contraste syntaxique qui, selon 
nous, rend possible la reprise par le pronom elle. De son côté, l'extrait (16) montre que le pronom personnel a la capacité de recatégoriser le référent.

\subsection{Les situations de reprise immédiate}

Examinons maintenant un autre type d'insertions discursives qui ont une parenté au moins formelle avec les situations qui nous occupent ici. Nous resituerons rapidement la problématique de la reprise immédiate en utilisant deux exemples habituellement proposés pour expliquer la concurrence des reprises anaphoriques définie et démonstrative en français :

(17) J'ai vu une voiture. Cette voiture roulait vite.

(18) J'ai vu une voiture et un camion. La voiture roulait vite.

En (17), P1 ne présentant qu'un seul antécédent, la reprise s'effectue au moyen du syntagme nominal démonstratif et non d'un SN défini. En (18), en revanche, si le déterminant défini est là tout à fait acceptable, c'est parce qu'en P1 deux antécédents sont présents. Cette analyse est celle de la théorie des contrastes de Corblin $(1987,1995)$ : 1) le ne peut être le déterminant de la seconde mention qu'à condition que le contexte lui fournisse un syntagme nominal avec lequel il puisse exercer un contraste lexical. C'est précisément ce que lui fournit la coordination en rendant disponibles d'autres $\mathrm{N}$ qui n'appartiennent pas à la classe du $\mathrm{N}$ de reprise et motive la saisie d'un individu par opposition à d'autres individus et 2) le démonstratif ce est en revanche impossible quand il s'agit de reprendre un $\mathrm{N}$ qui est accompagné d'autres $\mathrm{N}$ car ce déterminant suppose l'extraction d'un objet parmi les autres éléments de la même classe.

Si l'on examine contrastivement l'énoncé qui suit :

(19) Le vent est, au moins en partie, à l'origine du dépôt des loss. Le lœss est un dépôt jaune, homogène, en principe non lité, non consolidé, assez cohérent cependant pour garder des abrupts verticaux. [Précis] 
on peut relever deux antécédents potentiels en P1 (le vent et des loess). Et pourtant, aucune opposition notionnelle ne nous semble pouvoir être invoquée.

Nos énoncés ne se conforment strictement pas à la distribution habituellement attendue. D'abord, parce que la première mention y est généralement précédée d'un déterminant indéfini et la seconde d'un déterminant défini. Et, ensuite, parce que la répétition ne se fait pas à l'identique puisque le syntagme nominal de reprise est un syntagme générique qui ne renvoie donc pas à un objet particulier mais à une catégorie d'objets particuliers. Un regard du côté des travaux qui se sont donnés pour objectif de préciser les raisons du choix de l'un ou l'autre de ces déterminants en situation de reprise immédiate nous permet néanmoins de mettre en lumière les spécificités des situations de reprise "définitoires".

Finalement, deux contraintes fortes pesant sur l'insertion discursive des structures étudiées ne peuvent être entièrement expliquées à partir des propositions antérieures. Il s'agit d'abord de la réitération du syntagme de première mention qui, on l'a vu, est un enchaînement proscrit et ensuite du mode de coréférence choisi étant entendu que celui qui est le plus attendu est le pronom personnel il (entravé par la présence en P2 d'un prédicat classifiant).

Aucune des analyses précédentes ne nous fournissant à elles seules les moyens d'expliquer la reprise définie fidèle massive que nous observons, nous allons avancer une hypothèse explicative. L'objectif de notre second volet sera donc d'expliquer le maintien du syntagme de première mention alors que toutes les conditions d'une reprise pronominale démonstrative sont remplies. Nous admettons qu'un contraste est nécessaire, mais nous n'argumentons ni pour un contraste lexical ni pour un contraste notionnel mais pour un contraste d'un autre type, comme nous allons essayer de le montrer. 


\section{Un contraste autonymique}

Les situations qui font l'objet de notre étude faisant intervenir les relations entre phrases, il est difficile de se fier à la seule intuition pour juger des enchaînements possibles et (in)acceptables. Pour cette raison, nous utilisons des portions de texte extraites d'un corpus. Nous appuyant sur les observations de Combettes \& Tomassone (1988), nous tirons principalement nos extraits de textes explicatifs dont ces auteurs ont montré qu'ils forment un genre propice aux enchaînements qui nous occupent ici. Nos observations portent finalement sur une cinquantaine de contextes similaires.

\subsection{Arguments pour une absence de coréférence}

Les formes de reprise observées dans les textes sont ou bien la reprise $\mathrm{du} \mathrm{SN}$ de première mention ou bien le pronom démonstratif ou enfin il s'agit. Ce qui semble imposer la redénomination fidèle, ainsi que les deux autres formes de reprise, et, par conséquent, exclure la reprise pronominale, cela semble bien être le prédicat classifiant qui suit. Deux facteurs favorables se rencontrent : le fait que le syntagme de reprise est un syntagme générique et qu'on a affaire au prédicat classifiant "être un". L'examen des tours concurrents nous sera utile pour relever certaines contraintes qui pèsent sur les expressions employées et qui jouent un rôle dans le choix de l'anaphore. Pour ce faire comparons les deux extraits suivants :

(20) Gravitation : attraction de la matière par la matière. C'est une force universelle qui ne souffre aucune opposition. (in Combettes \& Tomassone, 1988: 97).

(19) Le vent est, au moins en partie, à l'origine du dépôt des loss. Le lœess est un dépôt jaune, homogène, en principe non lité, non consolidé, assez cohérent cependant pour garder des abrupts verticaux. [Précis]

Un aspect nous frappe immédiatement : on a deux antécédents potentiels en P1. La reprise pronominale par il est donc bloquée non seulement, comme le montre Kleiber (1994), parce que le prédicat est un prédicat classifiant, mais 
également parce qu'une reprise pronominale serait préférentiellement interprétée comme reprenant le SN sujet de P1, comme le prédit le Centrage. Il faut donc ajouter cette contrainte à celle du prédicat pour expliquer ces reprises.

Par contre, le seul argument du prédicat classifiant ne peut suffire à expliquer des énoncés non définitionnels comme celui qui suit :

(21) La silice peut aussi tapisser de multiples cavités superficielles de dissolution et former ainsi la meulière. La meulière provient d'une dissolution de calcaire et du dépôt immédiat de la silice sur la paroi de cavités de dissolution. [Précis]

L'explication de l'impossibilité d'une anaphore par il ne peut se satisfaire du seul argument de la présence d'un prédicat classifiant en P2. Le facteur qui nous semble déterminant ici est bien plutôt le fait qu'une anaphore pronominale serait préférentiellement interprétée comme renvoyant au SN topique en P1, à savoir "la silice". Ainsi n'a-t-on d'autres possibilités pour reprendre l'élément en focus dans $\mathrm{P} 1$ que de réintroduire le référent sous la forme de la première mention (de même une anaphore infidèle est exclue). Il semble donc que pour pouvoir être repris par un pronom dans un énoncé classifiant, Na doit occuper, dans P1, un statut spécial, autrement dit il doit être présenté comme un $\mathrm{N}$ nouveau.

Cet argument nous le trouvons chez Lambrecht (1994) qui pose que si on veut dire quelque chose de SN2, on ne peut se passer de le réintroduire dans le discours, de le réactiver comme topique. C'est ce que montre l'extrait :

(22) L'existence des mouvements isostatiques empêche de faire des fjords des auges glaciaires submergées par une montée des eaux, puisque le mouvement ascensionnel du continent a par endroits dépassé l'amplitude de la remontée du plan d'eau. Les fjords sont dus au creusement de la glace au-dessous du zéro marin, fait qui n'a rien d'étonnant si l'on remarque que, la densité de la glace étant voisine de 0,9 , le glacier peut enfoncer, dans une eau de densité peu supérieure à 1 , des $9 / 10$ de son volume. Un glacier épais de $1000 \mathrm{~m}$ peut exercer une pression sur le fond jusqu'à $900 \mathrm{~m}$ de profondeur. Les fjords sont des auges creusées par un glacier de vallée sur le fond de la mer et dont la profondeur a pu 
être diminuée par un relèvement isostatique dépassant l'amplitude du relèvement eustatique du niveau marin. [Précis]

Le terme à définir, fjord, constitue le topique du discours. En tant que foyer d'attraction, il est présent dans l'ensemble du segment et s'accompagne d'une forte saillance cognitive. Il est repris une première fois sous la forme définie fidèle (les fjords) avant de l'être de nouveau dans le segment définitoire (les fjords). La reprise définie fidèle s'impose en seconde mention dans l'énoncé définitoire car, entre la seconde mention et l'énoncé définitoire, un autre topique (un glacier) est introduit. L'article défini générique produit le même effet que précédemment, mais ici c'est le contraste entre deux topiques co-présents qui permet à les fjords de remplir son rôle anaphorique.

Nous soutenons finalement qu'il n'y a pas coréférence entre les deux mentions de Na. Cette position s'appuie sur les trois indices suivants : 1) l'inaptitude du pronom il à reprendre le référent de P1 expliquée par la présence du prédicat classifiant en $\mathrm{P} 2,2$ ) le déterminant de première mention n'est pas obligatoirement le même que celui de seconde mention (on observe en effet un passage à la fois de l'article indéfini au défini mais aussi d'un article pluriel à un article singulier) et 3) la réitération du $\mathrm{N}$ de première mention est également un indice qui montre l'absence de coréférence. Sur le plan sémantique, l'effet produit est un effet de rupture dans la continuité du discours précédent, on a le sentiment d'un décrochage. P2 n'est pas la suite de P1, il se situe sur un autre plan.

Par conséquent, notre position s'éloigne maintenant de celle avancée par Kleiber (1994) qui considère qu'il existe une identité de référent en P1 et en $\mathrm{P} 2$. Notre position est la suivante. Comme lui nous pensons que la déviance de $i l$ s'explique par le prédicat classifiant, mais nous pensons aussi qu'il n'y a pas d'identité entre les deux référents. Nous le montrerons en argumentant pour une lecture autonymique de l'élément repris en P2. Nous faisons en effet l'hypothèse que s'il s'agissait véritablement du même référent, il ne serait pas exclu. S'il l'est c'est bien parce que le référent a changé, il ne s'agit plus du référent spécifique de première mention car le point de vue a 
changé. Nous posons donc que la continuité est assurée superficiellement mais cela masque une rupture que nous analysons comme une rupture autonymique.

A l'appui de notre hypothèse explicative nous citerons un extrait qui présente un passage dans lequel la structure se trouve dans une position particulière puisqu'elle figure entre parenthèses :

(23) La dissolution peut se faire en profondeur par l'action des eaux souterraines et en particulier par celle de la nappe phréatique (la nappe phréatique, du grec phrear, puits, est une nappe souterraine continue, celle qu'atteignent les puits). [Précis]

On peut finalement distinguer deux stratégies discursives offertes au scripteur lorsqu'il introduit un nouveau référent dont il veut fournir une description définitoire :

- soit il souhaite établir le nouveau référent dans le discours et dans ce cas, une fois qu'il l'a introduit en position de focus, il le reprend dans la description définitoire qui sert en même temps à l'installer comme nouveau topique du discours : c'est ce que montre l'extrait élargi de (19) :

(19') Sur les nappes alluviales, le vent exerce une action de déflation comme celle qui donne naissance aux regs désertiques. [...] Le vent est, au moins en partie, à l'origine du dépôt des loess. Le loess est un dépôt jaune, homogène, en principe non lité, non consolidé, assez cohérent cependant pour garder des abrupts verticaux. Relativement homométrique, avec des grains de l'ordre de 10 à 50 microns, il est constitué de grains de quartz et de feldspath, de divers minéraux (les minéraux lourds renseignant sur la provenance, proche ou lointaine suivant le cas, des matériaux constituants) et d'une proportion de calcaire allant en général de 5 à 30 p 100 (mais très faible dans les loess normands), présence qui est tout à fait énigmatique. Les loess se rencontrent, sous des épaisseurs de l'ordre de quelques mètres et plus rarement de quelques dizaines de mètres [...] [Précis]

- soit il ne souhaite pas installer le référent nouvellement introduit dans le discours et dans ce cas la prédication définitoire sera fournie entre parenthèses, celles-ci marquant 
l'insertion d'un élément détaché et isolé par rapport à la phrase.

Ces deux éléments nous semblent accréditer la thèse de Lambrecht (1994: 82 in Grobet, 2002) selon laquelle un élément ne peut devenir le topique que s'il a préalablement été introduit dans le discours. Cette conception de la continuité informationnelle nous semble apte à rendre compte de ce qu'on observe. Si le scripteur ne souhaite pas utiliser un référent présenté comme nouveau dans la suite de son discours, il ne faut pas qu'il prédique quelque chose (comme par exemple un énoncé définitoire) à son propos, il fera donc figurer l'énoncé dans un site clairement décroché du reste du discours parenthétique ou en note, par exemple. En revanche, une fois introduit comme nouveau topique il le maintient, comme on l'a vu ci-dessus.

\subsection{L'explication autonymique}

Pour expliquer la reprise définie fidèle dans une situation où la reprise par un démonstratif lui semble préférable, nous nous appuierons sur la propriété - mise en évidence par Riegel (1987, 1990) - qu'ont les énoncés correspondant au schéma syntaxique $[\mathrm{SNa}$ est un $\mathrm{Nx}-\mathrm{X}]$ de véhiculer simultanément deux types d'informations: une définition dite traditionnellement de chose et une définition métalinguistique. Selon l'auteur, rappelons-le, coexistent une interprétation littérale du schéma [ $\mathrm{SNa}$ est un $\mathrm{Nx}-\mathrm{X}]$ et une interprétation métalinguistique qui est dérivée de la précédente au terme d'un acte de langage indirect en vertu du fait que si habituellement, dans ces schémas phrastiques, les signes s'effacent devant leurs référents, leur versant dénominatif reste toutefois activé.

(24) Une section se reconnaît par le fait qu'elle est composée de blocs de texte (les paragraphes) situés sous un titre. Le titre est un bloc court isolé et typographié dans une fonte plus grasse, éventuellement souligné et numéroté. [IC]

En (24), nous dirons donc que, lors de la première introduction du référent sous le substantif titre, le signe titre se trouve "en usage", en revanche, lors de sa reprise fidèle, le signe se trouve "en mention" et cela 
bien que la structure de l'énoncé soit superficiellement référentielle. Dans cet exemple, le syntagme le titre renvoie à la catégorie référentielle qui lui est conventionnellement associée, et, par un acte de langage indirect fondé sur la réflexivité des signes linguistiques, le titre renvoie également au mot titre. Pour le dire autrement, à l'interprétation référentielle directe qui permet l'identification de l'objet "titre", s'ajoute une instruction sur le sens du mot titre. L'interprétation définitoire de l'énoncé repose donc sur une interprétation du signe "en mention", en tant que forme linguistique, comme le montrent les paraphrases suivantes :

(24') Le mot titre désigne un bloc court isolé et typographié dans une fonte plus grasse, éventuellement souligné et numéroté.

(24") On appelle titre un bloc court isolé et typographié dans une fonte plus grasse, éventuellement souligné et numéroté.

L'énoncé définitoire indirect marque le retour réflexif qu'opère le scripteur sur son dire. Après avoir introduit un mot nouveau dans un énoncé référentiel où ce terme est en usage, il le reprend, en mention cette fois, dans l'énoncé définitoire, pour préciser le sens de ce mot.

Le contraste qui rend possible la reprise immédiate du référent de première mention est ici remplacé par un contraste que l'on pourrait dire "autonymique". La reprise définie fidèle semble ainsi trouver une explication dans ce site inattendu. Il ne s'agit pas d'une stricte reprise du syntagme indéfini de première mention mais d'un versant seulement de celui-ci, son versant autonymique.

L'étude discursive des énoncés correspondant au schéma phrastique [SNa est un $\mathrm{Nx}-\mathrm{X}]$, nous semble ainsi apporter un nouvel argument à la thèse pragma-sémantique proposée par Riegel puisqu'une explication fondée sur une double lecture apparaît clairement rendre compte de la reprise définie fidèle dans un site dont elle est normalement exclue.

\subsubsection{Une explication en termes de condition d'aliénation}

A l'explication contrastive de la reprise immédiate proposée par divers auteurs, Theissen (1997) ajoute une interprétation fondée sur la notion de 
condition d'aliénation. Elle montre que pour être repris adéquatement sous la forme fidèle, le référent doit avoir acquis une forme d'autonomie. L'auteur complète l'analyse des situations de coordination à l'appui de la notion de condition d'aliénation proposée par Kleiber (1999: 85). Rappelons-en brièvement le principe. Le propos de Kleiber, dans cet article, est d'établir la différence sémantique qui sépare une anaphore associative en Le $N$ de SN (le tronc $d u$ vieux tilleul) d'une anaphore réduite à Le $N$ (le tronc) dans l'exemple de Fradin (1984: 65) :

(25) Il s'abrita sous un vieux tilleul. Le tronc était tout craquelé.

Selon Kleiber l'anaphore associative s'explique par le fait que le référent du syntagme le tronc s'est libéré du prédicat de la phrase où il a été introduit. Si l'individu reste dépendant de la mention antérieure, le tronc du vieux tilleul, l'article défini, le, lui confère une forme de liberté. Il est vrai que la partie n'est pas effectivement disjointe du tout, mais elle s'en trouve isolée ou détachée; elle est appréhendée de façon autonome. L'hypothèse qu'il formule ainsi est que l'anaphore associative nécessite que l'individu soit autonome, "aliéné", par rapport à l'individu de l'expression antécédente.

Theissen explique que la difficulté qu'il y a à interpréter le syntagme défini, le $N$, en situation de reprise immédiate, provient du fait que le référent du SN sous le couvert duquel le syntagme de reprise a été introduit n'est pas autonome de son prédicat de première mention. Ce que montre ainsi l'auteur, c'est que l'aliénation du référent par rapport au prédicat favorise la reprise définie fidèle. Lorsque le référent du $\mathrm{SN}$ de première mention est immédiatement repris, il reste fortement dépendant de son prédicat de première mention ; or, la reprise définie fidèle nécessite, pour se réaliser, une aliénation du référent par rapport à son prédicat. La situation de coordination permet au référent de l'expression indéfinie de première mention de se libérer de son prédicat spécifiant et donc d'acquérir l'autonomie nécessaire à la reprise définie fidèle.

En dehors des situations de coordination, la reprise définie fidèle est ressentie comme peu naturelle lorsqu'elle est immédiate. Deux autres facteurs favorisent, selon Theissen (1997), l'aliénation nécessaire à cette forme de reprise. Le premier facteur qui favorise la reprise définie fidèle 
consiste en l'introduction d'un espace discursif, d'une distance discursive, qui permet au référent de se constituer en mémoire sous la forme d'un objet indépendant de son prédicat de première mention. L'autre solution consiste à choisir une reprise définie infidèle qui marque une distance permettant au référent d'acquérir son autonomie sous la forme de la montée hiérarchique.

Reprenons notre extrait (19) :

(19) Le vent est, au moins en partie, à l'origine du dépôt des loss. Le lœss est un dépôt jaune, homogène, en principe non lité, non consolidé, assez cohérent cependant pour garder des abrupts verticaux. [Précis]

En (19), les conditions d'un changement dénominatif décrites par Theissen (1997) sont remplies puisque le référent introduit par le syntagme indéfini de première mention est immédiatement repris et que la seconde mention du référent intervient sans qu'une séquence discursive de laquelle le référent serait absent soit insérée. On observe, néanmoins, un maintien du substantif qui a servi à introduire le référent dans le discours dans le syntagme défini anaphorique sans que l'interprétation référentielle soit entravée. Et cela s'explique selon nous par le fait qu'il ne s'agit pas du même référent.

On voit donc qu'un facteur qui favorise la reprise définie fidèle réside dans le fait que le référent du $\mathrm{SN}$ de première mention constitue une entité individuée, en vertu du principe d'aliénation. C'est le fait que le syntagme de reprise le loess est un syntagme générique qui permet de rendre le référent indépendant de son prédicat de première mention puisqu'il constitue un objet autonome. La distance textuelle nécessaire à l'acquisition de l'autonomie du référent est ici remplacée par une distance "générique" qui vient suppléer à l'absence de distance textuelle. Ce facteur nous semble s'accorder avec la lecture métalinguistique de l'énoncé que nous proposons de privilégier.

Par ailleurs, contrairement à ce qu'on aurait pu attendre, la variation typographique apparaît ici sur la première mention et non dans l'énoncé définitoire. Le gras confère au mot loess un statut ambigu: usage ou mention? On peut interpréter cet emploi comme une manière d'attirer l'attention du lecteur sur les mots employés dans un énoncé qui porte sur le monde. Le gras est ici un moyen de signaler le statut autonymique du mot 
nouveau introduit par le scripteur. On reconnaît là la connotation autonymique qui, rappelons-le, désigne le cumul d'une référence au monde et d'une référence au signe (cf. Rey-Debove, 1978).

L'indice métalinguistique, ici le gras, annonce l'énoncé qui va suivre et oriente l'interprétation vers le mot. C'est ce qui nous conduit à considérer que les énoncés que nous étudions se présentent comme des réponses aux questions potentielles d'un lecteur qui, au terme de la phrase où un nouveau référent est introduit dans le discours, pourrait demander qu'est-ce que c'est qu'un lœss? ou ce que vous appelez lœss, qu'est-ce que c'est? Ces énoncés semblent ainsi constituer des lieux du discours où le locuteur veut attirer l'attention de son lecteur, comme s'il anticipait la question d'un interlocuteur potentiel sur le code qu'il utilise. Aussi interpréterons-nous le rôle de la reprise comme un rôle instructionnel, au sens où elle signale un changement de perspective, le passage à un énoncé qui ne porte plus sur la chose mais sur la classe, et de façon indirecte sur la dénomination elle-même. L'interprétation que nous proposons s'accorde donc avec le principe d'aliénation de Kleiber (1999).

Notre analyse de l'insertion discursive des énoncés définitoires copulatifs rejoint finalement celle proposée par Riegel $(1987,1990)$ qui fait de ces énoncés de structure $[\mathrm{SNa}$ est un $\mathrm{Nx}-\mathrm{X}]$ des énoncés définitoires indirects. Nous avons montré que ces énoncés opèrent une rupture dans le fil du discours référentiel et cela sans qu'aucune marque explicite ne signale le changement de perspective. Nous avons ainsi proposé d'analyser ce changement de point de vue énonciatif comme un contraste autonymique. L'ensemble des énoncés définitoires servant à accomplir le même acte de langage, ils sont tous potentiellement équivalents dans le discours. On pourrait maintenant se demander ce qui conduit le scripteur à sélectionner un énoncé définitoire indirect, alors que d'autres formes explicites sont disponibles. Mais cela fera sans doute l'objet d'un autre article. 


\section{Références}

Combettes, B. \& Tomassone, R. (1988). Le texte informatif, aspects linguistiques. Bruxelles: De Boeck Université.

Corblin, F. (1987). Indéfini, défini et démonstratif. Genève : Droz.

Corblin, F. (1995). Les formes de reprise dans le discours: Anaphores et chaînes de référence. Rennes : Presses Universitaires de Rennes.

Cornish, F. (1999). Anaphora, Discourse and Understanding. Evidence from English and French. Oxford: Clarendon Press.

Cornish, F. (2000). L'accessibilité cognitive des référents, le Centrage d'attention et la structuration du discours : une vue d'ensemble. Verbum, 22 (1), pp. 7-30.

Cornish, F. (2001). L'inversion "locative" en français, italien et anglais : propriétés syntaxiques, sémantiques et discursives. Cahiers de grammaire (26), pp. 101-123.

Fradin, B. (1984). Anaphorisation et stéréotypes nominaux. Lingua, 64, 325369.

Grobet, A. (2002). L'identification des topiques dans les dialogues. Bruxelles: Duculot.

Kleiber, G. (1994). Anaphores et pronoms. Louvain La neuve: Duculot.

Kleiber, G. (1999). Anaphore associative et relation partie-tout : condition d'alinéation et principe de congruence ontologique. Langue française, 122, 70-100.

Rebeyrolle, J. (2000a). Utilisation de contextes définitoires pour l'acquisition de connaissances à partir de textes, Journées Francophones d'Ingénierie des Connaissances, IC'2000 (pp. 105-114), Toulouse, mai 2000.

Rebeyrolle, J. (2000b). Forme et fonction de la définition en discours. Doctorat Nouveau Régime, Université de Toulouse II.

Rey-Debove, J. (1978). Le métalangage. Paris : Le Robert.

Riegel, M. (1987). Définition directe et indirecte dans le langage ordinaire : les énoncés définitoires copulatifs. Langue française, 73, 29-53.

Riegel, M. (1990). La définition, acte du langage ordinaire - De la forme aux interprétations. In J. Chaurand \& F. Mazière (eds.), La définition, pp.97-110, Paris : Larousse.

Riegel, M., Pellat, J.-C., Rioul, R. (1994). Grammaire méthodique du français. Paris : Presses Universitaires de France.

Schnedecker, C. (2003). La question du nom propre répété dans la théorie dite du centrage et ses problèmes. Journal of French Language Studies, 13, 105-134.

Theissen, A. (1997). Le choix du nom en discours. Genève-Paris : Librairie Droz. 
Walker, M., Joshi, A. \& Prince, E. (1998). Centering Theory in Discourse. Oxford: Clarendon Press. 
Résumé : Cet article porte sur les énoncés définitoires indirects et se donne pour objectif de préciser comment se marque l'intention définitoire du locuteur dans ces énoncés qui ne comportent aucun indicateur explicite de leur force illocutionnaire. L'analyse de l'insertion discursive de ces énoncés conduit à mettre en lumière le fait que les situations, dans lesquelles le terme à définir fait l'objet d'une première mention généralement immédiate, sont favorables pour marquer le passage du discours référentiel au discours définitoire. L'explication tient compte de la structure informationnelle des énoncés et propose une analyse en terme de décrochage autonymique. 